\title{
VALUES OF HARMONIC WEAK MAASS FORMS ON HECKE ORBITS
}

\author{
DOHOON CHOI, MIN LEE, AND SUBONG LIM
}

\begin{abstract}
Let $q:=e^{2 \pi i z}$, where $z \in \mathbb{H}$. For an even integer $k$, let $f(z):=q^{h} \prod_{m=1}^{\infty}\left(1-q^{m}\right)^{c(m)}$ be a meromorphic modular form of weight $k$ on $\Gamma_{0}(N)$. For a positive integer $m$, let $T_{m}$ be the $m$ th Hecke operator and $D$ be a divisor of a modular curve with level $N$. Both subjects, the exponents $c(m)$ of a modular form and the distribution of the points in the support of $T_{m} . D$, have been widely investigated.

When the level $N$ is one, Bruinier, Kohnen, and Ono obtained, in terms of the values of $j$ invariant function, identities between the exponents $c(m)$ of a modular form and the points in the support of $T_{m} . D$. In this paper, we extend this result to general $\Gamma_{0}(N)$ in terms of values of harmonic weak Maass forms of weight 0 . By the distribution of Hecke points, this applies to obtain an asymptotic behaviour of convolutions of sums of divisors of an integer and sums of exponents of a modular form.
\end{abstract}

\section{INTRODUCTION}

Let $\mathbb{H}$ be the complex upper half plane. For a positive integer $N$, let $Y_{0}(N)$ be the modular curve of level $N$ defined by $\Gamma_{0}(N) \backslash \mathbb{H}$, and $X_{0}(N)$ denote the compactification of $Y_{0}(N)$ by adjoining the cusps. Let $J_{0}(N)$ be the jacobian of a modular curve $X_{0}(N)$. We denote by $\operatorname{Div}(C)$ the divisor group of a curve $C$. If $f$ is a function on $C$ and $D=\sum_{P \in C} n_{P} P$ is a divisor of $C$, we define

$$
f(D):=\sum n_{P} f(P) .
$$

The $m$ th normalized Hecke operator $T_{m}$ acts on $\operatorname{Div}\left(Y_{0}(N)\right)$, and it is denoted by $T_{n} . D$ for $D \in$ $\operatorname{Div}\left(Y_{0}(N)\right)$. We call $T_{m}$.D the $m$ th Hecke orbit of $D$. Especially, when $D$ is a divisor corresponding to $i \in \mathbb{H}$, a point in the support of $T_{m} . D$ is called a Hecke point. Hecke points have been investigated from several perspectives such as their distribution on the fundamental domain for $\Gamma_{0}(N)$ [13, 14, 17, 16] and the rank of a subgroup of $J_{0}(N)$ generated by Hecke points [21], and so on. Let $q:=e^{2 \pi i z}$, where $z \in \mathbb{H}$. For an even integer $k$, let $f(z):=q^{h} \prod_{m=1}^{\infty}\left(1-q^{m}\right)^{c(m)}$ be a meromorphic modular form of weight $k$ on $\Gamma_{0}(N)$. The exponents $c(m)$ of a modular form were investigated in various works (for examples, see [4, 5, 23]). For example, Borcherds [5] proved that if $f$ has a Heegner divisor, then the $m$ th exponent $c(m)$ is the $m^{2}$ th coefficient of a fixed modular form of half integral weight. Bruinier, Kohnen, and Ono [9] obtained a connection between these exponents of a modular form and the points in the support of $T_{n} . D$.

2010 Mathematics Subject Classification. 11F25, 11F12.

D. Choi was supported by Samsung Science and Technology Foundation under Project SSTF-BA1301-11. M. Lee was partially supported by Royal Society University Research Fellowship "Automorphic forms, L-functions and trace formulas".

Keywords: Hecke orbits, harmonic weak Maass forms, distribution. 
For the modular invariant $j$, let $J(z):=j(z)-744$. For positive integers $k$ and $m$, let $\sigma_{k}(m):=$ $\sum_{d \mid m} d^{k}$, and $\sigma_{f}(m):=\sum_{d \mid m} c(d)$. Bruinier, Kohnen, and Ono [9] proved the following identities between values $J\left(T_{m} . D_{f}\right)$ and sum of exponents in the product expansion of $f$ :

$$
\sum_{d \mid m} c(d) d=2 k \sigma_{1}(m)+J\left(T_{m} . D_{f}\right)
$$

for every positive integer $m$, where $D_{f}$ denotes the divisor of $f$ on $X_{0}(N)$. In other words, the value $J\left(T_{m} . D_{f}\right)$ can be expressed as the sum of the following values:

(1) a multiple of the divisor function $\sigma_{1}(m)$,

(2) the convolution of $\sigma_{1}(m)$ (sum of divisors) and $\sigma_{f}(m)$ (sum of exponents).

They applied this result to prove the modularity of the generating series for $\sigma_{f}(m)$ and to obtain several $p$-adic properties of $J\left(T_{m} . D_{f}\right)$ and exponents of a meromorphic modular form $f$. Based on the argument in [9], the result was extended to several cases such as $\Gamma_{0}(N)$ with genus zero by Ahlgren [2], Jacobi forms by Choie and Kohnen [12], and higher levels by the first author [11].

For general positive integers $N$, the first author studied in [11] the generalization of [9] to a harmonic weak Maass form $J_{N, 1}$ of weight 0 defined as a Poincaré series (instead of a weakly holomorphic modular form of weight 0$)$. It was proved in [11 that the value $J_{N, 1}\left(T_{m}\right.$. $\left.D_{f}\right)$ can be expressed as the sum of the following values:

(1) a linear combination of the divisor functions $\sigma_{1}(\mathrm{~nm})$ for $n \mid N$,

(2) the convolution of $\sigma_{1}(m)$ (sum of divisors) and $\sigma_{f}(m)$ (sum of exponents),

(3) the regularized Petersson inner product $R_{f, N}(m)$ of a meromorphic modular form and a cusp form.

In this paper, we show that $R_{f, N}(m)$, the value of the regularized Petersson inner product in identities [11], is zero, and so we give explicit identities between values $J_{N, 1}\left(T_{m} \cdot D_{f}\right)$ and sums of exponents in the product expansion of $f$. As an application, we obtain an asymptotic behavior for the convolution of $\sigma_{1}(m)$ (sum of divisors) and $\sigma_{f}(m)$ (sum of exponents) as $m \rightarrow \infty$.

Recently, Bringmann, Kane, Löbrich, Ono, and Rolen [7] showed that for any fixed $N$ the generating series for $J_{N, 1}\left(T_{m} . D_{f}\right)$ is basically modular. Moreover, their result implies that there is a cusp form such that, for each $m, R_{f, N}(m)$, the value of regularized Petersson inner product, is given by the $m$ th coefficient of a fixed cusp form.

Let $\mathcal{F}_{1}$ denote the usual fundamental domain for the action of $\mathrm{SL}_{2}(\mathbb{Z})$ on $\mathbb{H}$ given by

$$
\mathcal{F}_{1}:=\left\{z \in \mathbb{H}|| z \mid>1,-\frac{1}{2} \leq \operatorname{Re}(z)<\frac{1}{2}\right\} \cup\{z \in \mathbb{H}|| z \mid=1, \operatorname{Re}(z) \leq 0\}
$$

and

$$
\mathcal{F}_{N}:=\bigcup_{\gamma \in \mathrm{SL}_{2}(\mathbb{Z}) \backslash \Gamma_{0}(N)} \gamma \mathcal{F}_{1} .
$$

Here we choose coset representatives for $\mathrm{SL}_{2}(\mathbb{Z}) \backslash \Gamma_{0}(N)$ such that

$$
\mathcal{F}_{N} \subset\left\{z \in \mathbb{H}|| \operatorname{Re}(z) \mid \leq \frac{1}{2}\right\} \text {. }
$$

Then, $\mathcal{F}_{N}$ is a fundamental domain for the action of $\Gamma_{0}(N)$ on $\mathbb{H}$. Let $\mathcal{C}_{N}$ be the set of inequivalent cusps of $\Gamma_{0}(N)$. Let $k$ be an even integer and $f$ be a meromorphic modular form of weight $k$ on 
$\Gamma_{0}(N)$. For $\tau \in \mathbb{H} \cup\{i \infty\} \cup \mathbb{Q}$, let $Q_{\tau}$ be the image of $\tau$ under the canonical map from $\mathbb{H} \cup\{i \infty\} \cup \mathbb{Q}$ to $X_{0}(N)$. For $\tau \in \mathbb{H} \cup\{i \infty\} \cup \mathbb{Q}$, we denote by $\nu_{\tau}^{(N)}(f)$ the order of zero of $f$ at $Q_{\tau}$ on $X_{0}(N)$. Let us note

$$
g\left(T_{m} \cdot D_{f}\right)=\sum_{\tau \in \mathcal{F}_{N}} \nu_{\tau}^{(N)}(f) g\left(T_{m} \cdot \tau\right)
$$

Moreover, for a divisor $D=\sum n_{z} Q_{z}$ of $X_{0}(N)$, we can give a more explicit expression of $T_{m} . D$. For $\gamma=\left(\begin{array}{ll}a & b \\ c & d\end{array}\right) \in \mathrm{GL}_{2}(\mathbb{R})$ with positive determinant, we define the action of $\gamma$ for $z \in \mathbb{H}$ by

$$
\gamma z:=\frac{a z+b}{c z+d}
$$

For a positive integer $m$ prime to $N$, let

$$
T(m):=\left\{\gamma=\left(\begin{array}{ll}
a & b \\
0 & d
\end{array}\right) \mid a, b, d \in \mathbb{Z}, a>0, a d=m, \text { and } 0 \leq b<d\right\} .
$$

Then, we have

$$
T_{m} \cdot D=\sum n_{z} \sum_{\gamma \in T(m)} Q_{\gamma z}
$$

Next, we define the Ramanujan theta-operator by

$$
\theta(f)(z):=\frac{1}{2 \pi i} \frac{d}{d z} f(z)
$$

Let

$$
f_{\theta}(z):=\frac{\theta f(z)}{f(z)}-\frac{k}{12} E_{2}(z),
$$

where $E_{2}$ is the usual normalized Eisenstein series of weight 2 for $\mathrm{SL}_{2}(\mathbb{Z})$.

Let $N>1$ and $I_{v}$ be the usual modified Bessel functions as in [1]. For a positive integer $n$, we define the Poincaré series of weight 0 and index $n$ by

$$
F_{N, n}(z, s):=\sum_{\gamma \in \Gamma_{0}(N)_{\infty} \backslash \Gamma_{0}(N)} \pi|n \operatorname{Im}(\gamma z)|^{1 / 2} I_{s-\frac{1}{2}}(|2 \pi n \operatorname{Im}(\gamma z)|) e(-n \operatorname{Re}(\gamma z)),
$$

where $s \in \mathbb{C}$ with $\operatorname{Re}(s)>1$ and $e(z):=e^{2 \pi i z}$. Let $j_{N, n}(z)$ be the continuation of $F_{N, n}(z, s)$ as $s \rightarrow 1$ from the right. Then, the function $j_{N, n}$ is a harmonic weak Maass form of weight 0 on $\Gamma_{0}(N)$ (see [11, Section 2] for details). Let $J_{N, n}(z):=j_{N, n}(z)-\beta_{N, n}$, where $\beta_{N, n}$ is the constant term of the Fourier expansion of $j_{N, n}$ at the cusp $i \infty$.

For square-free $N$, let $D(N)$ be the number of divisors of $N$, and $\left\{d_{1}, d_{2}, \ldots, d_{D(N)-1}, N\right\}$ be the set of distinct divisors of $N$ such that $d_{i_{1}}<d_{i_{2}}$ if $i_{1}<i_{2}$. Let $A_{N}$ be the $(D(N)-1) \times(D(N)-1)$ matrix whose $i j$-entry $a_{i j}$ is defined by

$$
a_{i j}=\left(1-\frac{\operatorname{gcd}\left(d_{i}, d_{j}\right)^{2}}{d_{j}}\right)
$$

Let $A_{f, j}$ be a matrix obtained from $A_{N}$ by replacing the $j$ th column of $A_{N}$ with a column matrix whose $i$ th component is $\nu_{1 / d_{i}}^{(N)}(f)-\frac{k}{12}$. With this notation, we state our main theorem. 
Theorem 1.1. Let $k$ be an even integer and $N>1$ be a positive integer. Suppose that

$$
f(z)=q^{h_{\infty}} \prod_{n=1}^{\infty}\left(1-q^{n}\right)^{c(n)}
$$

is a meromorphic modular form of weight $k$ on $\Gamma_{0}(N)$. Then

$$
-\sum_{\tau \in \mathcal{F}_{N}} \nu_{\tau}^{(N)}(f)-\sum_{m=1}^{\infty}\left(\sum_{\tau \in \mathcal{F}_{N}} \nu_{\tau}^{(N)}(f) J_{N, m}(\tau)\right) q^{m}=f_{\theta}(z)-\mathcal{E}_{2}(z),
$$

where $\mathcal{E}_{2}$ is a modular form in the Eisenstein space of weight 2 on $\Gamma_{0}(N)$. Moreover, if $N$ is square free, then, for every positive integer $m$ prime to $N$,

$$
-J_{N, 1}\left(T_{m} \cdot D_{f}\right)=\sum_{d \mid m} d c(d)+24\left(\sum_{1 \leq j \leq D(N)-1} \frac{\operatorname{det}\left(A_{f, j}\right)}{\operatorname{det}\left(A_{N}\right)}+\frac{k}{12}\right) \sigma_{1}(m) .
$$

Remark 1.2. The modular form $\mathcal{E}_{2}$ in Theorem 1.1 is determined by the order of zero or pole of $f$ at each cusp. In many cases, a modular form $\mathcal{E}_{2}$ can be expressed as a sum of explicit modular forms. For example, if $N$ is square free, then

$$
\mathcal{E}_{2}(z)=\sum_{1 \leq j \leq D(N)-1} \frac{\operatorname{det}\left(A_{f, j}\right)}{\operatorname{det}\left(A_{N}\right)}\left(E_{2}(z)-d_{j} E_{2}\left(d_{j} z\right)\right) .
$$

Let $D:=\sum_{z \in S} n_{z} Q_{z}$ be a divisor of $Y_{0}(N)$, where $S$ is a finite set in $\mathcal{F}_{N}$. For a positive real number $r \geq 1$, we define a divisor $D_{>r}$ by

$$
D_{>r}=\sum_{\substack{z \in S \\ \operatorname{Im}(\tilde{z})>r}} n_{z} Q_{\tilde{z}}
$$

Here, $\tilde{z}$ is a complex number in $\mathcal{F}_{N}$, which is equivalent to $z$ under the action of $\Gamma_{0}(N)$. By the argument of Duke [15] and equidistribution of Hecke points ([17], [13] and [14]), Theorem [1.1] implies the following theorem.

Theorem 1.3. Let $k, N$, and $f$ be given as in Theorem 1.1. Assume that $N$ is square free. Let $m$ be a positive integer prime to $N$, and $h_{f}$ denote the sum of the orders of zero or pole of $f$ at $Q_{\tau}$ on $Y_{0}(N)$. Then

$$
\begin{aligned}
\lim _{m \rightarrow \infty} & \frac{1}{\sigma_{1}(m)}\left(24\left(\sum_{1 \leq j \leq D(N)-1} \frac{\operatorname{det}\left(A_{f, j}\right)}{\operatorname{det}\left(A_{N}\right)}+\frac{k}{12}\right) \sigma_{1}(m)-\sum_{d \mid m} d c(d)-e\left(-\left(T_{m} \cdot D_{f}\right)_{>1}\right)\right) \\
& =\frac{3 h_{f}}{\pi\left[\mathrm{SL}_{2}(\mathbb{Z}): \Gamma_{0}(N)\right]} \lim _{\epsilon \rightarrow 0} \int_{\mathcal{F}_{N}(\epsilon)} J_{N, 1}(z) \frac{d x d y}{y^{2}},
\end{aligned}
$$

where $c(n)$ are complex numbers determined by (1.1). Here, $\mathcal{F}_{N}(\epsilon)$ is defined by $\mathcal{F}_{N}-\cup_{\tau \in \mathcal{C}_{N}} B_{\tau}(\epsilon)$, where $B_{\tau}(\epsilon)$ is given in (3.1).

Recently, Ali and Mani [3] proved an upper bound for exponents $c(m)$ in the product expansion of $f$. The sum $\sum_{d \mid m} d c(d)$ looks like a kind of convolution of $\sigma_{1}(m)$ (a sum of divisors) and $\sigma_{f}(m$ ) 
(a sum of exponents of $f$ ). The above inequality means that, as $m \rightarrow \infty$, this convolution has a similar asymptotic behavior as that of the sum of divisors of $m$ except its main term.

The remainder of the paper is organized as follows. In Section 2, we introduce some preliminaries for meromorphic 1-forms on $X_{0}(N)$. In Section [3, we provide some basic facts on regularized Petersson inner product, and prove that $f_{\theta}$ is orthogonal to every cusp form of weight 2 on $\Gamma_{0}(N)$ with respect to regularized Petersson inner product if $f$ is a meromorphic modular form on $\Gamma_{0}(N)$. In Section 4, we recall some results related to the distribution of Hecke points for $\Gamma_{0}(N)$. In Section 5, we prove our main theorems: Theorems 1.1 and 1.3 .

\section{Residues of A MEROMORPhiC 1-Form on $X_{0}(N)$}

Let $f$ be a meromorphic modular form of weight 2 on $\Gamma_{0}(N)$. Assume that $t$ is a cusp of $\Gamma_{0}(N)$. Let $\sigma_{t} \in \mathrm{SL}_{2}(\mathbb{Z})$ be a matrix such that $\sigma_{t}(i \infty)=t$, and $\Gamma_{0}(N)_{t}$ denote the stabilizer of the cusp $t$ in $\Gamma_{0}(N)$. We define a positive integer $\alpha_{t}$ by

$$
\sigma_{t}^{-1} \Gamma_{0}(N)_{t} \sigma_{t}=\left\{ \pm\left(\begin{array}{cc}
1 & \ell \alpha_{t} \\
0 & 1
\end{array}\right): \ell \in \mathbb{Z}\right\}
$$

and we call $\alpha_{t}$ the width of $\Gamma_{0}(N)$ at the cusp $t$. The Fourier expansion of $f$ at the cusp $t$ is given by

$$
\left(\left.f\right|_{2} \sigma_{t}\right)(z)=\sum a_{t}(n) q^{n / \alpha_{t}}
$$

where $\left.\right|_{k}$ denotes the usual weight $k$ slash operator. If a cusp $t$ is equivalent to $i \infty$, the Fourier coefficients $a_{t}(n)$ of $f$ at the cusp $t$ are simply denoted by $a(n)$.

For $\tau \in \mathbb{H} \cup\{i \infty\} \cup \mathbb{Q}$, let $Q_{\tau}$ be the image of $\tau$ under the canonical map from $\mathbb{H} \cup\{i \infty\} \cup \mathbb{Q}$ to $X_{0}(N)$. Then, $f d z$ can be considered as a meromorphic 1 -form on $X_{0}(N)$. Thus, we denote by $\operatorname{Res}_{Q_{\tau}} f d z$ the residue of $f$ at $Q_{\tau}$ on $X_{0}(N)$. Let $\operatorname{Res}_{\tau} f$ be the residue of $f$ at $\tau$ on $\mathbb{H}$. The description of $\operatorname{Res}_{Q_{\tau}} f d z$ is given in terms of $\operatorname{Res}_{\tau} f$. For $\tau \in \mathbb{H}$, let $e_{\tau}$ be the order of the isotropy subgroup of $\Gamma_{0}(N)$ at $\tau$. Then, we have

$$
\operatorname{Res}_{Q_{\tau}} f d z= \begin{cases}\frac{1}{e_{\tau}} \operatorname{Res}_{\tau} f, & \text { if } \tau \in \mathbb{H}, \\ \frac{1}{2 \pi i} \alpha_{\tau} a_{\tau}(0), & \text { if } \tau \in \mathcal{C}_{N} .\end{cases}
$$

Let us note that if $k$ is an even integer and $f$ is a meromorphic modular form of weight $k$ on $\Gamma_{0}(N)$, then $f_{\theta}$ is a meromorphic modular form of weight 2 on $\Gamma_{0}(N)$. The residue of $f_{\theta}$ at each point on $X_{0}(N)$ is determined by the order of its zero or pole of $f$ at that point. Let $\operatorname{ord}_{\tau}(f)$ be the order of the zero or pole of $f$ at $\tau$ on $\mathbb{H}$. Since we have

$$
(c z+d)^{-2} E_{2}\left(\frac{a z+b}{c z+d}\right)=E_{2}(z)+\frac{12}{2 \pi i} \cdot \frac{c}{c z+d}
$$

for all $\left(\begin{array}{ll}a & b \\ c & d\end{array}\right) \in \mathrm{SL}_{2}(\mathbb{Z})$, we obtain

$$
\left(\left.f_{\theta}\right|_{2} \sigma_{t}\right)(z)=\frac{\theta\left(\left.f\right|_{k} \sigma_{t}\right)(z)}{\left(\left.f\right|_{k} \sigma_{t}\right)(z)}-\frac{k}{12} E_{2}(z)
$$

for a cusp $t$. Thus, we have

$$
\operatorname{Res}_{Q_{\tau}} f_{\theta} d z= \begin{cases}\frac{1}{2 \pi i} \nu_{\tau}^{(N)}(f) & \text { if } \tau \in \mathbb{H} \\ \frac{\alpha_{\tau}}{2 \pi i}\left(\nu_{\tau}^{(N)}(f)-\frac{k}{12}\right) & \text { if } \tau \in \mathcal{C}_{N}\end{cases}
$$




\section{Regularized Petersson inner product}

Petersson defined an inner product of two cusp forms with the same weight. The Petersson inner product was extended by Borcherds [6] to the case in which one of the two forms is a weakly holomorphic modular form. In this section, following [6] and [11, we define regularized Petersson inner product of a cusp form and a meromorphic modular form with the same weight. We prove that if $f$ is a meromorphic modular form on $\Gamma_{0}(N)$, then the regularized Petersson inner product of $f_{\theta}$ with any cusp form of weight 2 on $\Gamma_{0}(N)$ is zero.

Let $k$ be an even integer and $f$ be a meromorphic modular form of weight $k$ on $\Gamma_{0}(N)$. Let $\operatorname{Sing}(f)$ be the set of singular points of $f$ on $\mathcal{F}_{N}$. For a positive real number $\varepsilon$, an $\varepsilon$-disk $B_{\tau}(\varepsilon)$ at $\tau$ is defined by

$$
B_{\tau}(\varepsilon):= \begin{cases}\{z \in \mathbb{H}:|z-\tau|<\varepsilon\}, & \text { if } \tau \in \mathbb{H} \\ \left\{z \in \mathcal{F}_{N}: \operatorname{Im}\left(\sigma_{\tau} z\right)>1 / \varepsilon\right\}, & \text { if } \tau \in\{i \infty\} \cup \mathbb{Q} .\end{cases}
$$

Let $\mathcal{F}_{N}(f, \varepsilon)$ be a punctured fundamental domain for $\Gamma_{0}(N)$ defined by

$$
\mathcal{F}_{N}(f, \varepsilon):=\mathcal{F}_{N}-\bigcup_{\tau \in \operatorname{Sing}(f) \cup \mathcal{C}_{N}} B_{\tau}(\varepsilon)
$$

Let $g$ be a cusp form of weight $k$ on $\Gamma_{0}(N)$. The regularized Petersson inner product $(f, g)_{\text {reg }}$ of $f$ and $g$ is defined by

$$
(f, g)_{\text {reg }}:=\lim _{\varepsilon \rightarrow 0} \int_{\mathcal{F}_{N}(f, \varepsilon)} f(z) \overline{g(z)} \frac{d x d y}{y^{k-2}} .
$$

Then, we have the following proposition.

Proposition 3.1. Let $k$ be an even integer, and $f$ be a meromorphic modular form of weight $k$ on $\Gamma_{0}(N)$. Then, for every cusp form $g$ of weight 2 on $\Gamma_{0}(N)$,

$$
\left(f_{\theta}, g\right)_{\text {reg }}=0 \text {. }
$$

Proof. Let $\Delta(z):=q \prod_{n=1}^{\infty}\left(1-q^{n}\right)^{24}$ be the unique normalized cusp form of weight 12 on $\mathrm{SL}_{2}(\mathbb{Z})$. Let

$$
F(z):=\frac{f(z)^{12}}{\Delta(z)^{k}}
$$

Then, we have

$$
d\left(\left(\log _{e}|F(z)|^{2}\right) \overline{g(z)} d \bar{z}\right)=\frac{\partial_{z} F(z) \overline{F(z)}}{F(z) \overline{F(z)}} \overline{g(z)} d z d \bar{z}=\frac{\partial_{z} F(z)}{F(z)} \overline{g(z)}(-2 i) d x d y .
$$

Let us note that $\Delta$ has no zeros and no poles on $\mathbb{H}$. Therefore, according to [9, Theorem 1], we have

$$
\frac{\theta(\Delta)}{\Delta}=E_{2}
$$

The function $\partial_{z} F(z) / F(z)$ is given as

$$
\frac{\partial_{z} F(z)}{F(z)}=12 \frac{\partial_{z} f(z)}{f(z)}-k \frac{\partial_{z} \Delta(z)}{\Delta(z)}=12 \frac{\partial_{z} f(z)}{f(z)}-k(2 \pi i) E_{2}(z)=(24 \pi i) f_{\theta}(z) .
$$


Thus, we have

$$
d\left(\left(\log _{e}|F(z)|^{2}\right) \overline{g(z)} d \bar{z}\right)=(48 \pi) f_{\theta}(z) \overline{g(z)} d x d y
$$

In order to apply the Stokes theorem, we describe the boundary of $\mathcal{F}_{N}$. For a positive real number $\varepsilon$, we define

$$
\gamma_{\tau}(\varepsilon):= \begin{cases}\{z \in \mathbb{H}:|z-\tau|=\varepsilon\} & \text { if } \tau \in \mathbb{H} \\ \left\{z \in \mathcal{F}_{N}: \operatorname{Im}\left(\sigma_{\tau} z\right)=1 / \varepsilon\right\} & \text { if } \tau \in\{i \infty\} \cup \mathbb{Q} .\end{cases}
$$

Assume that $\varepsilon$ is sufficiently small. If $\partial^{*} \mathcal{F}_{N}(f, \varepsilon)$ denotes the closure of the set $\partial \mathcal{F}_{N}(f, \varepsilon)-\partial \mathcal{F}_{N}$ in $\mathbb{C}$, then

$$
\partial^{*} \mathcal{F}_{N}(f, \varepsilon)=\bigcup_{\tau \in \operatorname{Sing}(f) \cup \mathcal{C}_{N}} \gamma_{\tau}(\varepsilon),
$$

where $\partial D$ denotes the boundary of $D$ for a subset $D$ of $\mathbb{C}$. From (3.2) and (3.3), the Stokes theorem implies

$$
\begin{aligned}
\int_{\mathcal{F}_{N}\left(f_{\theta}, \varepsilon\right)} f_{\theta}(z) \overline{g(z)} d x d y & =\int_{\partial^{*} \mathcal{F}_{N}\left(f_{\theta}, \varepsilon\right)} \frac{1}{48 \pi}\left(\log _{e}|F(z)|^{2}\right) \overline{g(z)} d \bar{z} \\
& =\sum_{\tau \in \operatorname{Sing}\left(f_{\theta}\right) \cup \mathcal{C}_{N}} \int_{\gamma_{\tau}(\varepsilon)} \frac{1}{48 \pi}\left(\log _{e}|F(z)|^{2}\right) \overline{g(z)} d \bar{z}
\end{aligned}
$$

For each $\gamma \in \mathrm{SL}_{2}(\mathbb{Z})$, the absolute value $\left|\left(\left.g\right|_{2} \gamma\right)(z)\right|$ exponentially decays as $\operatorname{Im}(z) \rightarrow \infty$, since $g$ is a cusp form. Thus, if $\tau \in \mathcal{C}_{N}$, then $\lim _{\varepsilon \rightarrow 0} \int_{\gamma_{\tau}(\varepsilon)} \frac{1}{48 \pi}\left(\log _{e}|F(z)|^{2}\right) \overline{g(z)} d \bar{z}=0$.

To complete the proof, we assume that $\tau \in \operatorname{Sing}\left(f_{\theta}\right)$. Then

$$
\begin{aligned}
& \left|\int_{\gamma_{\tau}(\varepsilon)} \frac{1}{48 \pi}\left(\log _{e}|F(z)|^{2}\right) \overline{g(z)} d \bar{z}\right| \\
& \leq \int_{\gamma_{\tau}(\varepsilon)} \frac{1}{48 \pi}\left|\left(\log _{e}|F(z)|^{2}\right)\right||\overline{g(z)}||d \bar{z}| \\
& \left.\leq \max \left\{\left|\left(\log _{e}|F(z)|^{2}\right)\right|: z \in \gamma_{\tau}(\varepsilon)\right\} M_{1} \int_{\gamma_{\tau}(\varepsilon)}|d \bar{z}| \quad \text { some constant } M_{1}\right) \\
& \leq \max \left\{\left|\left(\log _{e}|F(z)|^{2}\right)\right|: z \in \gamma_{\tau}(\varepsilon)\right\} M_{1}(2 \pi \varepsilon) .
\end{aligned}
$$

The function $F(z)$ can be expressed around $\tau$ as

$$
F(z)=(z-\tau)^{12 \nu_{\tau}^{(N)}(f)} F_{0}(z)
$$

where $F_{0}(z)$ is a nowhere vanishing holomorphic function around $\tau$. If $\varepsilon$ is sufficiently small, then, for any $z \in \gamma_{\tau}(\varepsilon)$ we have

$$
\begin{aligned}
\left|\left(\log _{e}|F(z)|^{2}\right)\right| & \leq\left|\left(\log _{e}\left|\left(z-z_{0}\right)\right|^{24 \nu_{\tau}^{(N)}(f)}\right)\right|+\left|\left(\log _{e}\left|F_{0}(z)\right|^{2}\right)\right| \\
& \left.\leq\left|\left(\log _{e}\left|\left(z-z_{0}\right)\right|^{24 \nu_{\tau}^{(N)}(f)}\right)\right|+M_{2} \quad \text { some fixed constant } M_{2}\right) \\
& =\left|24 \nu_{\tau}^{(N)}(f) \log _{e} \varepsilon\right|+M_{2} .
\end{aligned}
$$


Thus, for sufficiently small $\varepsilon$, we have

$$
\left|\int_{\gamma_{\tau}(\varepsilon)} \frac{1}{48 \pi}\left(\log _{e}|F(z)|^{2}\right) \overline{g(z)} d \bar{z}\right| \leq\left(\left|24 \nu_{\tau}^{(N)}(f) \log _{e} \varepsilon\right|+M_{2}\right) M_{1}(2 \pi \varepsilon) .
$$

This implies that, for $\tau \in \operatorname{Sing}\left(f_{\theta}\right)$,

$$
\lim _{\varepsilon \rightarrow 0} \int_{\gamma_{\tau}(\varepsilon)} \frac{1}{48 \pi}\left(\log _{e}|F(z)|^{2}\right) \overline{g(z)} d \bar{z}=0 .
$$

Thus, we complete the proof.

\section{Equidistribution of Hecke points}

Let $\left\{u_{j}\right\}_{j \geq 0}$ be an orthonormal basis of the residual and cuspidal spaces of $L^{2}\left(\Gamma_{0}(N) \backslash \mathbb{H}\right)$, i.e., $u_{0}$ is a constant with the eigenvalue $\lambda_{0}=0$ and $u_{j}$ is a Maass form for $\Gamma_{0}(N)$ with eigenvalue $\lambda_{j}=s_{j}\left(1-s_{j}\right)$ for $j \geq 1$. Further, assume that $\lambda_{j}$ are ordered so that $0<\lambda_{1} \leq \lambda_{2} \leq \cdots$. For each cusp $t \in \mathbb{Q} \cup\{\infty\}$, let $E_{t}(z, s)$ be the Eisenstein series at $t$ for $\operatorname{Re}(s)>1$, which is given by

$$
E_{t}(z, s)=\sum_{\gamma \in \Gamma_{0}(N)_{t} \backslash \Gamma_{0}(N)}\left(\operatorname{Im}\left(\sigma_{t}^{-1} \gamma z\right)\right)^{s} .
$$

Here, $\Gamma_{0}(N)_{t} \subset \Gamma_{0}(N)$ is the stability group of $t$. For the properties of $E_{t}(z, s)$, see [19, $\left.\S 15\right]$.

According to [19, Theorem 15.5], any $f \in L^{2}\left(\Gamma_{0}(N) \backslash \mathbb{H}\right)$ has the spectral decomposition

$$
f(z)=\sum_{j \geq 0}\left\langle f, u_{j}\right\rangle u_{j}(z)+\sum_{t \in \mathcal{C}_{N}} \frac{1}{4 \pi} \int_{\mathbb{R}}\left\langle f, E_{t}(*, 1 / 2+i r)\right\rangle E_{t}(z, 1 / 2+i r) d r
$$

(valid in $L^{2}$-sense) and converges absolutely and uniformly on compact sets if $f$ and $\Delta f$ are smooth and bounded.

We now follow the proof of [17, Theorem 3.1]. Let

$$
\begin{aligned}
& f_{C}=\left\langle f, u_{0}\right\rangle=\text { the projection of } f \text { onto the constant subspace, } \\
& f_{M}(z)=\sum_{j \geq 1}\left\langle f, u_{j}\right\rangle u_{j}(z), \\
& f_{E}(z)=\sum_{t \in \mathcal{C}_{N}} \frac{1}{4 \pi} \int_{\mathbb{R}}\left\langle f, E_{t}(*, 1 / 2+i r)\right\rangle E_{t}(z, 1 / 2+i r) d r .
\end{aligned}
$$

Note that

$$
f_{C}=\left\langle f, u_{0}\right\rangle=\int_{\mathcal{F}_{N}} f(z) d \mu(z)
$$

where $d \mu(z):=\frac{3}{\pi\left[\mathrm{SL}_{2}(\mathbb{Z}): \Gamma_{0}(N)\right]} \cdot \frac{d x d y}{y^{2}}$ is the normalized Haar measure; so, $\int_{\mathcal{F}_{N}} d \mu(z)=1$.

Let $\lambda_{j}(n)$ be the $n$th Fourier coefficient of $u_{j}$. By the Ramanujan conjecture, there exists $\theta \geq 0$ such that $\left|\lambda_{j}(n)\right| \leq c n^{\theta+\epsilon}$, for any $\epsilon>0$. So, we get

$$
\frac{1}{\sigma_{1}(n)}\left\|T_{n} f_{M}\right\|_{2} \leq c n^{-\frac{1}{2}+\theta+\epsilon}\left\|f_{M}\right\|_{2}
$$

Note that the value of $\theta$ has been lowered to $\frac{7}{64}$ by Kim and Sarnak [20, Appendix 2]. 
In [22, $\S 6, \S 7$ and $\S 8]$, an explicit change-of-basis formula between the Eisenstein series attached to cusps and newform Eisenstein series attached to pairs of primitive Dirichlet characters is described. The Eisenstein series attached to a Dirichlet character is an eigenfunction of Hecke operators $T_{n}$ for $\operatorname{gcd}(n, N)=1$, and the absolute values of the corresponding eigenvalues are bounded above by $\sigma_{0}(n) n^{-\frac{1}{2}}$. So, we get

$$
\frac{1}{\sigma_{1}(n)}\left\|T_{n} f_{E}\right\|_{2} \leq c n^{-\frac{1}{2}+\epsilon}\left\|f_{E}\right\|_{2}
$$

If we combine (4.1), (4.2), and (4.3), then we obtain the following theorem. For more general result, see [13].

Theorem 4.1. Let $f \in L^{2}\left(\Gamma_{0}(N) \backslash \mathbb{H}\right)$. For a positive integer $n$ prime to $N$, we have

$$
\left\|\frac{1}{\sigma_{1}(n)} T_{n} f-\int_{\mathcal{F}_{N}} f(z) d \mu(z)\right\|_{2} \leq c_{\epsilon} n^{-\frac{1}{2}+\theta+\epsilon}\|f\|_{2}
$$

for any $\epsilon>0$. The constant $c_{\epsilon}$ depends on $\epsilon$.

The pointwise convergence can be derived from [14, Proposition 8.2]. Note that elliptic differential operators are differential operators that generalize the Laplace-Beltrami operator $\Delta$. For an integer $m \geq 2$, assume that $f, \Delta^{m} f \in L^{2}\left(\Gamma_{0}(N) \backslash \mathbb{H}\right)$. Then, by [14, Proposition 8.2], for a compact subset $\omega \subset \mathcal{F}_{N}$, there exist constants $C_{1}(\omega)$ and $C_{2}(\omega)$ such that, for any $z_{0} \in \omega$

$$
\begin{aligned}
& \left|\frac{1}{\sigma_{1}(n)} T_{n} f\left(z_{0}\right)-\int_{\mathcal{F}_{N}} f(z) d \mu(z)\right| \\
& \quad \leq C_{1}(\omega)\left\|\frac{1}{\sigma_{1}(n)} T_{n} f-\int_{\mathcal{F}_{N}} f(z) d \mu(z)\right\|_{2}+C_{2}(\omega)\left\|\frac{1}{\sigma_{1}(n)} T_{n}\left(\Delta^{m} f\right)-\int_{\mathcal{F}_{N}}\left(\Delta^{m} f\right)(z) d \mu(z)\right\|_{2} .
\end{aligned}
$$

So, we have the following corollary.

Corollary 4.2. Assume that $f, \Delta^{2} f \in L^{2}\left(\Gamma_{0}(N) \backslash \mathbb{H}\right)$. Take a compact $\omega \subset \Gamma_{0}(N) \backslash \mathbb{H}$ and a positive number $\epsilon$. Then, there exists a constant $C_{\omega, \epsilon}$ depending on $\omega$ and $\epsilon$, such that, for a positive integer $n$ prime to $N$, for any $z_{0} \in \omega$,

$$
\left|\frac{1}{\sigma_{1}(n)} T_{n} f\left(z_{0}\right)-\int_{\mathcal{F}_{N}} f(z) d \mu(z)\right| \leq C_{\omega, \epsilon} n^{-\frac{1}{2}+\theta+\epsilon} \max \left\{\|f\|_{2},\left\|\Delta^{2} f\right\|_{2}\right\} .
$$

\section{Proofs}

Let $M_{k}^{\text {Eis }}\left(\Gamma_{0}(N)\right)$ be the space of modular forms orthogonal to all the cusp forms of weight $k$ on $\Gamma_{0}(N)$, which is called the Eisenstein space of weight $k$ on $\Gamma_{0}(N)$. In the following lemma, we prove that if $N$ is square-free, then, for a positive integer $n$ prime to $N$, the $n$th coefficient of a modular form in $M_{2}^{E i s}\left(\Gamma_{0}(N)\right)$ is a multiple of $\sigma_{1}(n)$. Recall the notations $D(N), d_{j}$, and $A_{N}$ from Section 1. Now, we prove the following lemma related to properties for modular forms in an Eisenstein space.

Lemma 5.1. Suppose that $\mathcal{E}_{2}(z):=\sum_{n=0}^{\infty} b(n) q^{n}$ is a modular form in $M_{2}^{\text {Eis }}\left(\Gamma_{0}(N)\right)$, and that $N$ is square free. Then, the following statements are true. 
(1) There exists a constant c such that for every positive integer $n$ prime to $N$,

$$
b(n)=c \sigma_{1}(n) .
$$

(2) Assume that the constant term of $\mathcal{E}_{2}(z)$ at cusp $1 / d_{i}$ is $c_{d_{i}}$. Let $A_{j}$ be the matrix obtained from $A$ by replacing the $j$ th column of $A$ with a column matrix whose ith component is $c_{d_{i}}$. Then

$$
\mathcal{E}_{2}(z)=\sum_{1 \leq j \leq D(N)-1} \frac{\operatorname{det}\left(A_{j}\right)}{\operatorname{det}\left(A_{N}\right)}\left(E_{2}(z)-d_{j} E_{2}\left(d_{j} z\right)\right) .
$$

Proof. (1) We claim that there is a basis of $M_{2}^{E i s}\left(\Gamma_{0}(N)\right)$ consisting of modular forms $E_{2}(z)-$ $d E_{2}(d z)$, where $d \neq 1$ are the divisors of $N$. Assume that the claim is true. Then, $\mathcal{E}_{2}(z)$ can be expressed as a linear combination of $E_{2}(z)-d_{j} E_{2}\left(d_{j} z\right)$ having the form

$$
\mathcal{E}_{2}(z)=\sum_{1 \leq j \leq D(N)-1} a_{j}\left(E_{2}(z)-d_{j} E_{2}\left(d_{j} z\right)\right) .
$$

Recall that $E_{2}$ has the Fourier expansion of the form

$$
E_{2}(z)=1-24 \sum_{n=1}^{\infty} \sigma_{1}(n) q^{n}
$$

Then, the $n$th coefficient of $\mathcal{E}_{2}(z)$ is given by

$$
-24 \sum_{1 \leq j \leq D(N)-1} a_{j}\left(\sigma_{1}(n)-d_{j} \sigma_{1}\left(n / d_{j}\right)\right)
$$

for $n>0$, and $a_{j}$ does not depend on $n$. Here, $\sigma_{1}(n / d)=0$ if $n$ is not divisible by $d$. Thus, we have the proof of the lemma.

Now, we prove the claim. Suppose that

$$
\sum_{1 \leq j \leq D(N)-1} a_{j}\left(E_{2}(z)-d_{j} E_{2}\left(d_{j} z\right)\right)=0
$$

where $a_{j}$ are complex numbers. We assume that complex numbers $a_{j}$ are not all zero. Then, we have

$$
\sum_{1 \leq j \leq D(N)-1} a_{j} E_{2}(z)=\sum_{1 \leq j \leq D(N)-1} a_{j} d_{j} E_{2}\left(d_{j} z\right)
$$

Comparing the $n$th coefficients of the forms on both sides for $n$ prime to $N$, we have

$$
\sum_{1 \leq j \leq D(N)-1} a_{j} E_{2}(z)=\sum_{1 \leq j \leq D(N)-1} a_{j} d_{j} E_{2}\left(d_{j} z\right)=0 .
$$

Take the smallest positive integer $d_{j_{0}} \mid N$ such that $a_{j_{0}} \neq 0$. Then, we have

$$
-a_{j_{0}} d_{j_{0}} E_{2}\left(d_{j_{0}} z\right)=\sum_{1 \leq j \leq D(N)-1} a_{j} d_{j} E_{2}\left(d_{j} z\right)-a_{j_{0}} d_{j_{0}} E_{2}\left(d_{j_{0}} z\right) \text {. }
$$

Comparing the $d_{j_{0}}$ th coefficients of the forms on both sides, we have $a_{j_{0}}=0$. This is a contradiction. Therefore, the modular forms $E_{2}(z)-d E_{2}(d z), d \mid N$ and $d \neq 1$, are linearly independent.

Let us note

$$
\operatorname{dim}_{\mathbb{C}} M_{2}^{E i s}\left(\Gamma_{0}(N)\right)=D(N)-1
$$


since $N$ is square free. Thus,

$$
\left\{\left(E_{2}(z)-d E_{2}(d z): d \mid N \text { and } d \neq 1\right\}\right.
$$

is a basis of $M_{2}^{\text {Eis }}\left(\Gamma_{0}(N)\right)$. This completes the proof of the claim.

(2) From the proof of (1), we may assume that

$$
\mathcal{E}_{2}(z)=\sum_{1 \leq j \leq D(N)-1} a_{j}\left(E_{2}(z)-d_{j} E_{2}\left(d_{j} z\right)\right) .
$$

Let us note that $E_{2}(z)-\frac{3}{\pi \operatorname{Im}(z)}$ is a non-holomorphic modular form of weight 2 on $\mathrm{SL}_{2}(\mathbb{Z})$. By direct computation, there are $\gamma \in \mathrm{SL}_{2}(\mathbb{Z})$ and $\mu_{j} \in \mathbb{Z}$ such that

$$
\left(\begin{array}{cc}
d_{j} & 0 \\
0 & 1
\end{array}\right)\left(\begin{array}{cc}
1 & 0 \\
d_{i} & 1
\end{array}\right)=\gamma\left(\begin{array}{cc}
1 & \mu_{j} \\
0 & d_{j} / \operatorname{gcd}\left(d_{j}, d_{i}\right)
\end{array}\right)\left(\begin{array}{cc}
\operatorname{gcd}\left(d_{j}, d_{i}\right) & 0 \\
0 & 1
\end{array}\right)
$$

Thus,

$$
\left.\left(E_{2}(z)-d_{j} E_{2}\left(d_{j} z\right)\right)\right|_{2}\left(\begin{array}{cc}
1 & 0 \\
d_{i} & 1
\end{array}\right)=E_{2}(z)-\frac{\operatorname{gcd}\left(d_{j}, d_{i}\right)^{2}}{d_{j}} E_{2}\left(\frac{\operatorname{gcd}\left(d_{j}, d_{i}\right)^{2}}{d_{j}} z+\frac{\mu_{j} \operatorname{gcd}\left(d_{j}, d_{i}\right)}{d_{j}}\right) .
$$

This implies that $a_{j}$ are the solution of the system

$$
c_{d_{i}}=\sum_{1 \leq j \leq D(N)-1}\left(1-\frac{\operatorname{gcd}\left(d_{j}, d_{i}\right)^{2}}{d_{j}}\right) a_{j}
$$

for $1 \leq i \leq D(N)-1$. Thus, the Cramer's rule completes the proof.

Now, we prove Theorem 1.1.

Proof. Note that

$$
\left\{E_{2}(z)-d E_{2}(d z)|d| N, d \neq 1\right\}
$$

forms a basis of $M_{2}^{E i s}\left(\Gamma_{0}(N)\right)$ by the proof of Lemma 5.1. Therefore, we can take a modular form $\mathcal{E}_{2} \in M_{2}^{E i s}\left(\Gamma_{0}(N)\right)$ such that the constant term of $\mathcal{E}_{2}$ at each cusp except cusps equivalent to $i \infty$ is the same as that of $f_{\theta}$. Suppose that $\mathcal{E}_{2}$ has the Fourier expansion of the form

$$
\mathcal{E}_{2}(z)=\sum_{n=0}^{\infty} b(n) q^{n}
$$

Note that, by (2.1) and (2.2), we have

$$
\frac{2 \pi i}{e_{\tau}} \operatorname{Res}_{\tau} f_{\theta}=\nu_{\tau}^{(N)}(f)
$$

for $\tau \in \mathcal{F}_{N}$. Thus, from [11, Lemma 3.1], we obtain

$$
\left(f_{\theta}-\mathcal{E}_{2}, \xi_{0}\left(j_{N, m}\right)\right)_{r e g}=\beta_{N, m}\left(a_{\theta}(0)-b(0)\right)+a_{\theta}(m)-b(m)+\sum_{\tau \in \mathcal{F}_{N}} \nu_{\tau}^{(N)}(f) j_{N, m}(\tau),
$$

where $a_{\theta}(m)$ is the $m$ th Fourier coefficient of $f_{\theta}$ and $\xi_{0}$ is a differential operator defined by

$$
\xi_{0}(f)(z):=2 i \overline{\frac{\partial}{\partial \bar{z}} f(z)} .
$$


By the same argument in the proof of [11, Lemma 3.1], we have

$$
\left(f_{\theta}-\mathcal{E}_{2}, \xi_{0}\left(J_{N, m}\right)\right)_{r e g}=a_{\theta}(m)-b(m)+\sum_{\tau \in \mathcal{F}_{N}} \nu_{\tau}^{(N)}(f) J_{N, m}(\tau) .
$$

Note that $\xi_{0}\left(j_{N, m}\right)=\xi_{0}\left(J_{N, m}\right)$ since $J_{N, m}(z)=j_{N, m}(z)-\beta_{N, m}$. Therefore, from (5.2) and (5.3), we have

$$
a_{\theta}(0)-b(0)=\frac{1}{\beta_{N, m}}\left(\sum_{\tau \in \mathcal{F}_{N}} \nu_{\tau}^{(N)}(f) J_{N, m}(\tau)-\sum_{\tau \in \mathcal{F}_{N}} \nu_{\tau}^{(N)}(f) j_{N, m}(\tau)\right)=-\sum_{\tau \in \mathcal{F}_{N}} \nu_{\tau}^{(N)}(f) .
$$

Proposition 3.1 implies that

$$
\left(f_{\theta}-\mathcal{E}_{2}, \xi_{0}\left(j_{N, m}\right)\right)_{\text {reg }}=0 .
$$

Therefore, from (5.3), we have

$$
a_{\theta}(m)-b(m)=-\sum_{\tau \in \mathcal{F}_{N}} \nu_{\tau}^{(N)}(f) J_{N, m}(\tau)
$$

for every positive integer $m$. Thus, from (5.4) and (5.5), we obtain

$$
f_{\theta}(z)-\mathcal{E}_{2}(z)=-\sum_{\tau \in \mathcal{F}_{N}} \nu_{\tau}^{(N)}(f)-\sum_{m=1}^{\infty}\left(\sum_{\tau \in \mathcal{F}_{N}} \nu_{\tau}^{(N)}(f) J_{N, m}(\tau)\right) q^{m} .
$$

By (1.1) and the Fourier expansion of $E_{2}$ given in (5.1), $f_{\theta}$ has the Fourier expansion of the form

$$
f_{\theta}(z)=h_{\infty}+\sum_{n=1}^{\infty} \sum_{d \mid n} d c(d) q^{n}-\frac{k}{12}+2 k \sum_{n=1}^{\infty} \sigma_{1}(n) q^{n} .
$$

Let us note that the constant term of $f_{\theta}$ at cusp $t$ is $\nu_{t}^{(N)}(f)-k / 12$. Suppose that $m$ is prime to $N$. Then, Lemma 5.1 implies that

$$
b(m)=-24\left(\sum_{1 \leq j \leq D(N)-1} \frac{\operatorname{det}\left(A_{f, j}\right)}{\operatorname{det}\left(A_{N}\right)}\right) \sigma_{1}(m) .
$$

Here, $A_{f, j}$ is a matrix obtained from $A_{N}$ by replacing the $j$ th column of $A_{N}$ with a column matrix whose $i$ th component is $\frac{\nu_{1 / d_{i}}^{(N)}(f)}{\alpha_{1 / d_{i}}}-\frac{k}{12}$. Let us note that if $\operatorname{gcd}(m, N)=1$, then $J_{N, m}=J_{N, 1} \mid T_{m}$. Therefore, by (5.5), (5.7), and (5.8), we have

$$
\begin{aligned}
-J_{N, 1}\left(T_{m} . D_{f}\right) & =-J_{N, m}\left(D_{f}\right)=-\sum_{\tau \in \mathcal{F}_{N}} \nu_{\tau}^{(N)}(f) J_{N, m}(\tau)=a_{\theta}(m)-b(m) \\
& =\sum_{d \mid m} d c(d)+24\left(\sum_{1 \leq j \leq D(N)-1} \frac{\operatorname{det}\left(A_{f, j}\right)}{\operatorname{det}\left(A_{N}\right)}+\frac{k}{12}\right) \sigma_{1}(m) .
\end{aligned}
$$


To prove Theorem 1.3, we follow the argument of the proof of [15, Proposition 3]. We fix $\epsilon>0$. Let $\psi_{\epsilon}: \mathbb{R}_{>0} \rightarrow \mathbb{R}$ be a $C^{\infty}$ function with $0 \leq \psi_{\epsilon}(y) \leq 1$ for all $y \in \mathbb{R}_{>0}$ and

$$
\psi_{\epsilon}(y)= \begin{cases}0, & \text { if } y \leq 1 \\ 1, & \text { if } y>1+\epsilon\end{cases}
$$

For a positive integer $n$, consider the Poincaré series defined by

$$
P_{n, \epsilon}(z):=\sum_{\gamma \in \Gamma_{0}(N)_{\infty} \backslash \Gamma_{0}(N)} \psi_{\epsilon}(\operatorname{Im}(\gamma z)) e(-n(\gamma z)) .
$$

From this, we obtain the following proposition.

Proposition 5.2. Let $\theta$ be given as in Section 4. Fix $n \in \mathbb{Z}_{\geq 1}, \epsilon>0$, and $z_{0} \in \mathbb{H}$. For any positive integer $m$ prime to $N$ and any $\epsilon^{\prime}>0$, we have

$$
\begin{array}{r}
\frac{1}{\sigma_{1}(m)}\left\{\sum_{\substack{a d=m, b \quad(\bmod d)}} J_{N, n}\left(\frac{a z_{0}+b}{d}\right)-\sum_{\begin{array}{c}
a d=m, \\
b \quad \bmod d)
\end{array}} P_{n, \epsilon}\left(\frac{a z_{0}+b}{d}\right)\right\}-\lim _{\epsilon^{\prime \prime} \rightarrow 0} \int_{\mathcal{F}_{N}\left(\epsilon^{\prime \prime}\right)} J_{N, n}(z) d \mu(z) \mid \\
\leq C_{z_{0}, \epsilon^{\prime}} m^{-\frac{1}{2}+\theta+\epsilon^{\prime}} \max \left\{\left\|F_{n, \epsilon}\right\|_{2},\left\|\Delta^{2} F_{n, \epsilon}\right\|_{2}\right\},
\end{array}
$$

where $F_{n, \epsilon}:=J_{N, n}-P_{n, \epsilon}$ and $C_{z_{0}, \epsilon^{\prime}}$ is the constant given as in Corollary 4.2.

Proof. For a positive integer $n$, let $P_{n, \epsilon}$ be the Poincaré series as in (5.9). From [11, Theorem 2.1], it follows that $F_{n, \epsilon} \in L^{2}\left(\Gamma_{0}(N) \backslash \mathbb{H}\right)$ for a fixed $n \geq 1$.

Recall that for $\phi \in L^{2}\left(\Gamma_{0}(N) \backslash \mathbb{H}\right)$ and $m \geq 1$ with $\operatorname{gcd}(m, N)=1$, the normalized Hecke operator $T_{m}$ can be represented by

$$
T_{m} \phi(z)=\sum_{\gamma \in T(m)} \phi(\gamma z)
$$

By Corollary 4.2, we find that for any $\epsilon^{\prime}>0$ and $m$

$$
\left|\frac{1}{\sigma_{1}(m)}\left(T_{m} F_{n, \epsilon}\right)\left(z_{0}\right)-\int_{\mathcal{F}_{N}} F_{n, \epsilon}(z) d \mu(z)\right| \leq C_{z_{0}, \epsilon^{\prime}} m^{-\frac{1}{2}+\epsilon^{\prime}+\theta} \max \left\{\left\|F_{n, \epsilon}\right\|_{2},\left\|\Delta^{2} F_{n, \epsilon}\right\|_{2}\right\} .
$$

For $z_{0} \in \mathbb{H}$, we have

$$
\frac{1}{\sigma_{1}(m)} T_{m} F_{n, \epsilon}\left(z_{0}\right)=\frac{1}{\sigma_{1}(m)}\left\{\sum_{\substack{a d=m, b \quad \bmod d)}} J_{N, n}\left(\frac{a z_{0}+b}{d}\right)-\sum_{\substack{a d=m, b \bmod d)}} P_{n, \epsilon}\left(\frac{a z_{0}+b}{d}\right)\right\} .
$$

Note that

$$
\lim _{\epsilon^{\prime \prime} \rightarrow 0} \int_{\mathcal{F}_{N}\left(\epsilon^{\prime \prime}\right)} P_{n, \epsilon}(z) \frac{d x d y}{y^{2}}=\int_{\Gamma_{0}(N)_{\infty} \backslash \mathbb{H}} \psi_{\epsilon}(y) e^{-2 \pi i n z} \frac{d x d y}{y^{2}}=\int_{0}^{\infty} \psi_{\epsilon}(y) e^{2 \pi n y} \frac{d y}{y^{2}} \cdot \int_{0}^{1} e^{2 \pi i n x} d x=0
$$

for every positive integer $n$. So, we get

$$
\int_{\mathcal{F}_{N}} F_{n, \epsilon}(z) d \mu(z)=\lim _{\epsilon^{\prime \prime} \rightarrow 0} \int_{\mathcal{F}_{N}\left(\epsilon^{\prime \prime}\right)} J_{N, n}(z) d \mu(z) .
$$


If we combine (5.10), (5.11), and (5.12), then we get the desired result.

We define

$$
Q_{1, \epsilon}(z):=\psi_{\epsilon}(\operatorname{Im}(\tilde{z})) e(-\tilde{z})
$$

for $\epsilon>0$. Then, we obtain the following proposition.

Proposition 5.3. For any $m$ with $\operatorname{gcd}(m, N)=1$, we obtain

$$
\begin{aligned}
\mid \frac{1}{\sigma_{1}(m)}\left(J_{N, 1}\left(T_{m} \cdot D_{f}\right)-Q_{1, \epsilon}\left(T_{m} . D_{f}\right)\right)- & h_{f} \lim _{\epsilon^{\prime \prime} \rightarrow 0} \int_{\mathcal{F}_{N}\left(\epsilon^{\prime \prime}\right)} J_{N, 1}(z) d \mu(z) \mid \\
& \leq H_{f} C\left(f, \epsilon^{\prime}\right) m^{-\frac{1}{2}+\theta+\epsilon^{\prime}} \max \left\{\left\|F_{1, \epsilon}\right\|_{2},\left\|\Delta^{2} F_{1, \epsilon}\right\|_{2}\right\},
\end{aligned}
$$

where $h_{f}$ denotes the sum of the orders of zero or pole of $f$ at $Q_{\tau}$ on $Y_{0}(N)$.

Proof. Let $\epsilon>0$ be fixed. Note that

$$
J_{N, 1}\left(T_{m} . D_{f}\right)=\sum_{\tau \in \mathcal{F}_{N}} \nu_{\tau}^{(N)}(f) \sum_{\substack{a d=m \\ b \\(\bmod d)}} J_{N, 1}\left(\frac{a \tau+b}{d}\right)
$$

and

$$
P_{1, \epsilon}\left(T_{m} . D_{f}\right)=\sum_{\tau \in \mathcal{F}_{N}} \nu_{\tau}^{(N)}(f) \sum_{\substack{a d=m, b \bmod d)}} P_{1, \epsilon}\left(\frac{a \tau+b}{d}\right) .
$$

Therefore, by Proposition 5.2 , for any $m$ with $\operatorname{gcd}(m, N)=1$, we have

$$
\begin{aligned}
\mid \frac{1}{\sigma_{1}(m)}\left(J_{N, 1}\left(T_{m} . D_{f}\right)-P_{1, \epsilon}\left(T_{m} . D_{f}\right)\right)- & h_{f} \lim _{\epsilon^{\prime \prime} \rightarrow 0} \int_{\mathcal{F}_{N}\left(\epsilon^{\prime \prime}\right)} J_{N, 1}(z) d \mu(z) \mid \\
& \leq H_{f} C\left(f, \epsilon^{\prime}\right) m^{-\frac{1}{2}+\theta+\epsilon^{\prime}} \max \left\{\left\|F_{1, \epsilon}\right\|_{2},\left\|\Delta^{2} F_{1, \epsilon}\right\|_{2}\right\},
\end{aligned}
$$

for any $\epsilon^{\prime}>0$, where $H_{f}:=\sum_{\tau \in \mathcal{F}_{N}}\left|\nu_{\tau}^{(N)}(f)\right|$ and $C\left(f, \epsilon^{\prime}\right):=\max \left\{C_{\tau, \epsilon^{\prime}} \mid \tau \in \mathcal{F}_{N}, \nu_{\tau}^{(N)}(f) \neq 0\right\}$.

Recall that $\tilde{z}$ is a unique complex number in $\mathcal{F}_{N}$ which is equivalent to $z$ under the action of $\Gamma_{0}(N)$. If $\operatorname{Im}(\tilde{z})>1$, then for any $\gamma \in \Gamma_{0}(N), \operatorname{Im}(\gamma \tilde{z}) \leq 1$ unless $\gamma \in \Gamma_{0}(N)_{\infty}$.

Suppose that $\operatorname{Im}(\tilde{z}) \leq 1$ and that there exists $\gamma \in \Gamma_{0}(N)$ such that $\operatorname{Im}(\gamma \tilde{z})>1$. Then, there exists $\ell \in \mathbb{Z}$ such that $-\frac{1}{2}<\operatorname{Re}(\gamma \tilde{z})+\ell \leq \frac{1}{2}$, and so

$$
\gamma \tilde{z}+\ell=\left(\begin{array}{ll}
1 & \ell \\
0 & 1
\end{array}\right) \gamma \tilde{z} \in \mathcal{F}_{N}
$$

Since $\left(\begin{array}{ll}1 & \ell \\ 0 & 1\end{array}\right) \gamma \in \Gamma_{0}(N)$, we have $\gamma \tilde{z}+\ell=\tilde{z}$, so $\operatorname{Im}(\gamma \tilde{z})=\operatorname{Im}(\tilde{z}) \leq 1$, which is a contradiction. Therefore, if $\operatorname{Im}(\tilde{z}) \leq 1$, then for any $\gamma \in \Gamma_{0}(N)$, we get $\operatorname{Im}(\gamma \tilde{z}) \leq 1$.

Thus, we have

$$
P_{1, \epsilon}(z)=P_{1, \epsilon}(\tilde{z})=\sum_{\gamma \in \Gamma_{0}(N)_{\infty} \backslash \Gamma_{0}(N)} \psi_{\epsilon}(\operatorname{Im}(\gamma \tilde{z})) e(-\gamma \tilde{z})=Q_{1, \epsilon}(z) .
$$

Therefore, from (5.14), we obtain the desired result. 
From Proposition 5.2 and Proposition 5.3, we obtain the following theorem. This gives the distribution of values of $J_{N, 1}$ on Hecke orbits.

Theorem 5.4. We have

$$
\lim _{m \rightarrow \infty} \frac{1}{\sigma_{1}(m)}\left(J_{N, 1}\left(T_{m} \cdot D_{f}\right)-e\left(\left(T_{m} \cdot D_{f}\right)_{>1}\right)\right)=\frac{3 h_{f}}{\pi\left[\mathrm{SL}_{2}(\mathbb{Z}): \Gamma_{0}(N)\right]} \lim _{\epsilon^{\prime \prime} \rightarrow 0} \int_{\mathcal{F}_{N}\left(\epsilon^{\prime \prime}\right)} J_{N, 1}(z) \frac{d x d y}{y^{2}} .
$$

Proof. Let $\epsilon>0$ be fixed. For any positive integer $m$ which is prime to $N$, we have

$$
\begin{aligned}
& \left|\frac{1}{\sigma_{1}(m)}\left(J_{N, 1}\left(T_{m} \cdot D_{f}\right)-e\left(\left(T_{m} \cdot D_{f}\right)_{>1}\right)\right)-h_{f} \lim _{\epsilon^{\prime \prime} \rightarrow 0} \int_{\mathcal{F}_{N}\left(\epsilon^{\prime \prime}\right)} J_{N, 1}(z) d \mu(z)\right| \\
& \leq\left|\frac{1}{\sigma_{1}(m)}\left(J_{N, 1}\left(T_{m} \cdot D_{f}\right)-Q_{1, \epsilon}\left(T_{m} \cdot D_{f}\right)\right)-h_{f} \lim _{\epsilon^{\prime \prime} \rightarrow 0} \int_{\mathcal{F}_{N}\left(\epsilon^{\prime \prime}\right)} J_{N, 1}(z) d \mu(z)\right| \\
& +\frac{1}{\sigma_{1}(m)}\left|Q_{1, \epsilon}\left(T_{m} \cdot D_{f}\right)-e\left(\left(T_{m} \cdot D_{f}\right)_{>1}\right)\right| .
\end{aligned}
$$

Note that

$$
\begin{aligned}
& \left|Q_{1, \epsilon}\left(T_{m} . D_{f}\right)-e\left(-\left(T_{m} . D_{f}\right)\right)_{>1}\right| \\
& \leq \sum_{\substack{\tau \in \mathcal{F}_{N} \\
\nu_{\tau}^{(N)}(f) \neq 0}}\left|\nu_{\tau}^{(N)}(f)\right| \sum_{\gamma \in T(m)} \begin{cases}\mid \psi_{\epsilon}(\operatorname{Im}(\widetilde{\gamma \tau})-1|| e(-\widetilde{\gamma \tau}) \mid, & \text { if } 1<\operatorname{Im}(\widetilde{\gamma \tau}) \leq 1+\epsilon, \\
0, & \text { otherwise. }\end{cases}
\end{aligned}
$$

Now, we follow the proof of [15, Proposition 3]. Fix $0<\epsilon<\frac{1}{4}$ and consider the incomplete Eisenstein series

$$
g_{\epsilon}(z):=\sum_{\gamma \in \Gamma_{0}(N)_{\infty} \backslash \Gamma_{0}(N)} \phi_{\epsilon}(\operatorname{Im} \gamma z)
$$

where $\phi_{\epsilon}: \mathbb{R}_{>0} \rightarrow \mathbb{R}$ is a smooth function supported in $(1-\epsilon, 1+2 \epsilon)$ with $0 \leq \phi_{\epsilon}(y) \leq 1$ for all $y \in \mathbb{R}_{>0}$ and $\phi_{\epsilon}(y)=1$ for $1 \leq y \leq 1+\epsilon$. By Corollary 4.2, we see that for any $\epsilon^{\prime}>0, z_{0} \in \mathbb{H}$, and $m$ with $\operatorname{gcd}(m, N)=1$,

$$
\left|\frac{1}{\sigma_{1}(m)} T_{m} g_{\epsilon}\left(z_{0}\right)-\int_{\mathcal{F}_{N}} g_{\epsilon}(z) d \mu(z)\right| \leq C_{z_{0}, \epsilon^{\prime}} m^{-\frac{1}{2}+\epsilon^{\prime}+\theta} \max \left\{\left\|g_{\epsilon}\right\|_{2},\left\|\Delta^{2} g_{\epsilon}\right\|_{2}\right\} .
$$

Then, there exists a constant $D\left(f, \epsilon^{\prime}\right)$ such that

$$
\begin{aligned}
& \frac{1}{\sigma_{1}(m)}\left|Q_{1, \epsilon}\left(T_{m} . D_{f}\right)-e\left(-\left(T_{m} . D_{f}\right)_{>1}\right)\right| \leq \frac{e^{2 \pi(1+\epsilon)}}{\sigma_{1}(m)} \sum_{\substack{\tau \in \mathcal{F}_{N} \\
\nu_{\tau}^{(N)}(f) \neq 0}}\left|\nu_{\tau}^{(N)}(f)\right|\left(T_{m} g_{\epsilon}\right)(\tau) \\
& \leq C_{f} e^{2 \pi(1+\epsilon)}\left(\int_{\mathcal{F}_{N}} g_{\epsilon}(z) d \mu(z)+D\left(f, \epsilon^{\prime}\right) m^{-\frac{1}{2}+\theta+\epsilon^{\prime}} \max \left\{\left\|g_{\epsilon}\right\|_{2},\left\|\Delta^{2} g_{\epsilon}\right\|_{2}\right\}\right),
\end{aligned}
$$

where $C_{f}:=\#\left\{\tau \in \mathcal{F}_{N} \mid \nu_{\tau}^{(N)}(f) \neq 0\right\} \times \max \left\{\left|\nu_{\tau}^{(N)}(f)\right| \mid \tau \in \mathcal{F}_{N}\right\}$. 
Therefore, from Proposition 5.3 and (5.15), we have

$$
\begin{aligned}
& \left|\frac{1}{\sigma_{1}(m)}\left(J_{N, 1}\left(T_{m} \cdot D_{f}\right)-e\left(\left(T_{m} \cdot D_{f}\right)>1\right)\right)-h_{f} \lim _{\epsilon^{\prime \prime} \rightarrow 0} \int_{\mathcal{F}_{N}\left(\epsilon^{\prime \prime}\right)} J_{N, 1}(z) d \mu(z)\right| \\
& \leq\left(H_{f} C\left(f, \epsilon^{\prime}\right) \max \left\{\left\|F_{1, \epsilon}\right\|_{2},\left\|\Delta^{2} F_{1, \epsilon}\right\|_{2}\right\}+C_{f} e^{2 \pi(1+\epsilon)} D\left(f, \epsilon^{\prime}\right) \max \left\{\left\|g_{\epsilon}\right\|_{2},\left\|\Delta^{2} g_{\epsilon}\right\|_{2}\right\}\right) m^{-\frac{1}{2}+\theta+\epsilon^{\prime}} \\
& \quad+C_{f} e^{2 \pi(1+\epsilon)} \int_{\mathcal{F}_{N}} g_{\epsilon}(z) d \mu(z) .
\end{aligned}
$$

For a fixed $\epsilon$, taking $m \rightarrow \infty$, we get

$$
\begin{aligned}
& \lim _{m \rightarrow \infty} \frac{1}{\sigma_{1}(m)}\left(J_{N, 1}\left(T_{m} \cdot D_{f}\right)-e\right.\left.\left(\left(T_{m} \cdot D_{f}\right)_{>1}\right)\right) \\
&=h_{f} \lim _{\epsilon^{\prime \prime} \rightarrow 0} \int_{\mathcal{F}_{N}\left(\epsilon^{\prime \prime}\right)} J_{N, 1}(z) d \mu(z)+C_{f} e^{2 \pi(1+\epsilon)} \int_{\mathcal{F}_{N}} g_{\epsilon}(z) d \mu(z) .
\end{aligned}
$$

Note that (5.19) holds for any fixed $0<\epsilon<\frac{1}{4}$. Since

$$
\int_{\mathcal{F}_{N}} g_{\epsilon}(z) d \mu(z)=\frac{3}{\pi\left[\mathrm{SL}_{2}(\mathbb{Z}): \Gamma_{0}(N)\right]} \int_{0}^{\infty} \phi_{\epsilon}(y) \frac{d y}{y^{2}} \rightarrow 0,
$$

as $\epsilon \rightarrow 0$, we get

$$
\lim _{m \rightarrow \infty} \frac{1}{\sigma_{1}(m)}\left(J_{N, 1}\left(T_{m} \cdot D_{f}\right)-e\left(\left(T_{m} . D_{f}\right)_{>1}\right)\right)=h_{f} \lim _{\epsilon^{\prime \prime} \rightarrow 0} \int_{\mathcal{F}_{N}\left(\epsilon^{\prime \prime}\right)} J_{N, 1}(z) d \mu(z) .
$$

Finally, Theorem 1.3 comes from Theorem 5.4 and (1.2).

\section{REFERENCES}

[1] M. Abramowitz and I. Stegun, Pocketbook of Mathematical Functions, Verlag Harri Deutsch, Thun, 1984.

[2] S. Ahlgren, The theta-operator and the divisors of modular forms on genus zero subgroups, Math. Res. Lett. 10 (2003), no. 5-6, 787-798.

[3] A. Ali and N. Mani, Infinite product exponents for modular forms, Res. Number Theory 2 (2016), Art. 21, 10 pp.

[4] R. E. Borcherds, Monstrous moonshine and monstrous Lie superalgebras, Invent. Math. 109, 405-444 (1992).

[5] R. E. Borcherds, Automorphic forms on $\mathcal{O}_{s+2,2}(\mathbb{R})$ and infinite products, Invent. Math. 120 (1995), no. 1, $161-213$.

[6] R. E. Borcherds, Automorphic forms with singularities on Grassmannians, Invent. Math. 132 (1998), no. 3, $491-562$.

[7] K. Bringmann, B. Kane, S. Löbrich, K. Ono, and L. Rolen, On divisors of modular forms, Adv. Math. 329 (2018), 541-554.

[8] J. H. Bruinier and J. Funke, On two geometric theta lifts, Duke Math. J. 125 (2004), no. 1, 45-90.

[9] J. H. Bruinier, W. Kohnen, and K. Ono, The arithmetic of the values of modular functions and the divisors of modular forms, Compos. Math. 140 (2004), no. 3, 552-566.

[10] D. Choi, On values of a modular form on $\Gamma_{0}(N)$, Acta Arith. 121 (2006), no. 4, 299-311.

[11] D. Choi, Poincaré series and the divisors of modular forms, Proc. Amer. Math. Soc. 138 (2010), no. 10, 3393-3403. 
[12] Y. Choie and W. Kohnen, Special values of elliptic functions at points of the divisors of Jacobi forms, Proc. Amer. Math. Soc. 131 (2003), no. 11, 3309-3317.

[13] L. Clozel, H. Oh, and E. Ullmo, Hecke operators and equidistribution of Hecke points, Invent. math. 144 (2001), 327-351.

[14] L. Clozel and E. Ullmo, Équidistribution des points de Hecke, Contributions to Automorphic Forms, Geometry, and Number Theory. Baltimore, MD: Johns Hopkins Univ. Press, 2004, 193-254.

[15] W. Duke, Modular functions and the uniform distribution of CM points, Math. Ann. 334 (2006), 241-252.

[16] W. T. Gan and H. Oh, Equidistribution of integer points on a family of homogeneous varieties: A problem of Linnik, Compos. Math. 136(3) (2003) 323-352.

[17] D. Goldstein and A. Mayer, On the equidistribution of Hecke points, Forum Mathematicum 15, no. 2 (2003): $165-189$.

[18] H. Iwaniec, Spectral methods of automorphic forms, Second edition, Graduate Studies in Mathematics, 53, American Mathematical Society, Providence, RI; Revista Matemtica Iberoamericana, Madrid, 2002.

[19] H. Iwaniec and E. Kowalski, Analytic number theory, American Mathematical Society Colloquium Publications 53, American Mathematical Society, Providence, RI, 2004, xii+615.

[20] H. Kim, Functoriality for the exterior square of $G L_{4}$ and the symmetric fourth of $G L_{2}$. With appendix 1 by Dinakar Ramakrishnan and appendix 2 by Kim and Peter Sarnak. J. Amer. Math. Soc. 16 (2003), no. 1, 139-183.

[21] J. Silverman, Hecke points on modular curves, Duke Math. J. 60 (1990), no. 2, 401-423.

[22] M. P. Young, Explicit calculations with Eisenstein series, preprint (arXiv:1710.03624).

[23] D. Zagier Traces of singular moduli, in: Motives, Polylogarithms and Hodge Theory, Part I, Int. Press Lect. Ser. 3, I, Int. Press, Somerville, MA, 2002, 211-244.

Department of Mathematics, Korea University, 145 Anam-ro, Seongbuk-gu, Seoul 02841, RePUBLIC OF KOREA

E-mail address: dohoonchoi@korea.ac.kr

Howard House, University of Bristol, Queens Ave, BS8 1SN, United Kingdom

E-mail address: min.lee@bristol.ac.uk

Department of Mathematics Education, Sungkyunkwan University, Jongno-gu, Seoul 03063, REPUBLIC OF KOREA

E-mail address: subong@skku.edu 